\title{
Efficient Data Optimisation for Harmonic Inpainting with Finite Elements ${ }^{\star}$
}

\author{
Vassillen Chizhov and Joachim Weickert \\ Mathematical Image Analysis Group, Faculty of Mathematics and Computer Science, \\ Campus E 1.7, Saarland University, 66041 Saarbrücken, Germany, \\ \{chizhov, weickert\}@mia.uni-saarland.de
}

\begin{abstract}
Harmonic inpainting with optimised data is very popular for inpainting-based image compression. We improve this approach in three important aspects. Firstly, we replace the standard finite differences discretisation by a finite element method with triangle elements. This does not only speed up inpainting and data selection, but even improves the reconstruction quality. Secondly, we propose highly efficient algorithms for spatial and tonal data optimisation that are several orders of magnitude faster than state-of-the-art methods. Last but not least, we show that our algorithms also allow working with very large images. This has previously been impractical due to the memory and runtime requirements of prior algorithms.
\end{abstract}

Keywords: Inpainting · Image reconstruction · Finite element method.

\section{Introduction}

In recent years, alternatives to transform-based compression have been proposed under the name inpainting-based compression; see e.g. [2, 6, 8, 9, 11, 13, 17, 19, 20 , 29. During encoding, these approaches store an optimised subset of the image data (e.g. $5 \%$ of all pixels), the so-called inpainting mask. In the decoding phase, they reconstruct an approximation of the original image from the mask data with the help of an inpainting process. Inpainting-based methods have been able to qualitatively outperform widely used codecs such as JPEG and JPEG2000; see e.g. 29. Two of the simplest inpainting methods are based on linear spline approximation over triangle meshes [1, 9, 10,22 and on discretisations of the Laplace equation 21] (also called homogeneous diffusion inpainting or harmonic inpainting) with the finite difference method (FDM). Although these approaches are relatively simple, they can achieve very good quality if the inpainting data

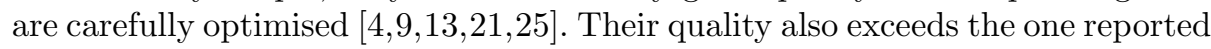
for recent neural network approaches for sparse inpainting [7].

Unfortunately, the data optimisation during the encoding phase is typically costly both in terms of memory and runtime. It consists of two problems: spatial

\footnotetext{
* This project has received funding from the European Research Council (ERC) under the European Union's Horizon 2020 research and innovation programme (grant agreement No 741215, ERC Advanced Grant INCOVID).
} 
optimisation and tonal optimisation. The spatial optimisation problem aims at finding the optimal locations on the image grid for the set of pixels to be stored. This is a hard combinatorial minimisation problem that requires efficient heuristics. In contrast, the tonal optimisation problem consists of modifying the grey values (or colour values) of the stored data. While this is a least squares problem, it can require large computational and memory resources $12,14,21$. This precludes previous tonal optimisation methods for harmonic inpainting from being applied to very large images.

Artifacts constitute another problem. Linear spline inpainting may suffer from conspicuous structures of the triangle mesh, and FDM-based harmonic inpainting typically exhibits logarithmic singularities at the stored pixels.

Our Contributions. The goal of our paper is to address the above-mentioned problems by introducing three improvements of high practical relevance:

1. We propose to implement harmonic inpainting with a finite element method (FEM) based on adaptive triangulation. Compared to finite differences on the pixel grid, its adaptivity allows to achieve better quality in lower time. Interestingly, this also alleviates the logarithmic singularities that are characteristic for the finite difference solution.

2. We devise a computationally efficient spatial optimisation strategy which scales better and is multiple orders of magnitude faster than current stateof-the-art approaches without compromising reconstruction quality.

3. We propose a computationally and memory efficient algorithm for tonal optimisation. Its runtime and memory requirements scale much more favourably with the image resolution compared to previous state-of-the-art approaches. This allows us to handle also very large images, which has been problematic for previous tonal optimisation approaches for harmonic inpainting.

Related Work. Finite elements have been successfully used for PDE models for image denoising $3,18,27$ and restoration 5,32 . However, to our knowledge they have not been applied to PDE-based image approximation from sparse data.

Various spatial optimisation strategies have been proposed for inpaintingbased compression. Mainberger et al. 21 introduced a probabilistic sparsification approach that selects a subset of pixels to be removed at each iteration. A subsequent nonlocal pixel exchange step relocates pixels probabilistically and keeps only relocations that decrease the error. The dithering-based densification strategy of Karos et al. 17] iteratively adds a fraction of the target pixels by halftoning an inpainting error image. The Voronoi densification of Daropoulos et al. 8 constructs a Voronoi diagram from the currently stored pixel data and inserts a new pixel in the cell with the highest error at each iteration. Our approach combines the ideas of densification with an error map 17 and a partitioning of the domain 8 . However, we use the tessellation already available from the FEM mesh. In our case this is a Delaunay triangulation which is dual to a Voronoi tessellation and avoids storing the mesh connectivity. 
Tonal optimisation approaches can be split into two categories: methods that explicitly compute the dense solution for the inpainting, and iterative descentbased strategies. The former ones include inpainting echoes 20], LSQR relying on an LU factorisation 14], and Green's functions [15. Their memory requirements scale quadratically in the number of mask points or image pixels. This makes them inapplicable for larger images. The second category includes gradient descent methods 12, 15], quasi-Newton techniques such as L-BFGS [6, and primal-dual algorithms 14]. Our approach falls in this category and is very efficient by using nested conjugate gradient iterations 28 .

Outline. In Section 2 we discuss the mathematical formulation of harmonic inpainting and the application of FEM to it. Then we describe our spatial optimisation approach in Section 3 and our tonal optimisation algorithm in Section 4. We present our results in Section 5 , and we conclude the paper with some potential avenues for future research in Section 6

\section{FEM for Harmonic Inpainting}

In this section we introduce the mathematical formulation for harmonic inpainting in the continuous setting and then motivate the choice of an FEM discretisation over an FDM discretisation. Additionally, we describe how the FEM mesh is constructed and how the solution is interpolated to all image pixels.

\subsection{Continuous Formulation of Harmonic Inpainting}

We use harmonic inpainting to construct an approximation of the original image given a sparse subset of the image data. Let us consider a continuous greyscale image $f: \Omega \rightarrow \mathbb{R}$ on some rectangular image domain $\Omega$. Rather than storing $f$ on the entire domain $\Omega$, we only specify values on a data domain $\mathrm{K} \subset \Omega$ (the so-called mask pixels in a discrete setting). In the remaining domain $\Omega \backslash \mathrm{K}$, we fill in missing values by solving the Laplace equation with reflecting boundary conditions on $\partial \Omega$ :

$$
\begin{aligned}
-\Delta u(\boldsymbol{x}) & =0, & & \boldsymbol{x} \in \Omega \backslash \mathrm{K}, \\
u(\boldsymbol{x}) & =g(\boldsymbol{x}), & & \boldsymbol{x} \in \mathrm{K}, \\
\partial_{\boldsymbol{n}} u(\boldsymbol{x}) & =0, & & \boldsymbol{x} \in \partial \Omega,
\end{aligned}
$$

where $\boldsymbol{n}$ is normal to $\partial \Omega$. In order to improve the approximation quality of $u$ w.r.t. $f$, one optimises the shape of the data domain $\mathrm{K}$ under some size constraint (spatial optimisation) and the corresponding grey values $g$ within $K$ (tonal optimisation). In the discrete setting, $\boldsymbol{f}, \boldsymbol{g}$, and $\boldsymbol{u}$ are vectors instead of functions.

\subsection{FEM Formulation}

Finite difference methods (FDM) 24] and finite element methods (FEM) 16] are two classes of numerical techniques used to solve differential equations. FDM is 
often applied on a regular and equidistant grid (the pixel grid for digital images), while FEM is well-suited for data on adaptive meshes. Therefore, the number of unknowns for the FDM method grows rapidly with the image resolution, while one has fine control over the number of unknowns in FEM by means of the construction of the underlying mesh. This flexibility naturally suggests using an adaptive lower resolution FEM mesh with fewer unknowns in order to speed up the inpainting compared to FDM.

Our FEM formulation relies on a triangle mesh; see Figure 2, right. Every mask pixel is a vertex in this mesh, and a subset of the remaining non-mask pixels are chosen as unknowns, depending on how many unknowns are required or on the runtime constraints. We call this subset the unknown vertices. If all non-mask pixels were chosen as unknown vertices, then the standard (5-point stencil) FDM discretisation of the Laplace equation would be recovered. Once the vertices for the mesh have been determined, a Delaunay triangulation is constructed from the point set formed by the vertices [26]. The Delaunay property of maximising the minimum angle in the triangulation is desirable, since it provides guarantees regarding the optimality of the resulting condition number for the matrices involved in the inpainting and tonal optimisation 30. Furthermore, this frees us from storing any connectivity, since the mesh can be reconstructed only from the mask pixels and unknown vertices 26 .

We consider a linear FEM method: The reconstructed image is linear within each triangle of the aforementioned mesh and continuous at edges and vertices. At each mask pixel the corresponding stored grey value is prescribed, while the values at the unknown vertices are found by solving the linear system arising from the FEM formulation. Each unknown corresponds to an unknown vertex. We typically choose as many unknown vertices as we have mask pixels. For further technical details on FEM, we refer to the standard literature [16]. Given a Delaunay mesh with at least one mask pixel, the system matrix is symmetric and positive definite. Thus, we can use the conjugate gradient method 28] in order to approximate the solution. Finally, the solution is linearly interpolated within each triangle for the remaining non-mask pixels which do not correspond to vertices in the mesh.

\section{Spatial Optimisation}

Our spatial optimisation algorithm is based on a novel coarse-to-fine error map densification strategy that combines concepts from an error map dithering 17 and a Voronoi densification algorithm 8 . The main idea is to introduce $m$ mask pixels over $n$ iterations, where each iteration requires a single inpainting.

To this end, we have investigated many strategies, and we now describe the one which has produced the best results. Most importantly, we have found that the error map must be considered at a locally adaptive scale that reflects the mesh structure as a function of the mask pixel density. In each iteration we introduce $\frac{m}{n}$ of all mask pixels. In the very first iteration we distribute all unknown vertices uniformly at random on the image grid, and introduce the 
first $\frac{m}{n}$ mask pixels also uniformly at random. In each subsequent iteration, we compute the inpainted image $\boldsymbol{u}$ from the current mesh, and its error map $e_{i}=\left(u_{i}-f_{i}\right)^{2}$ in each pixel $i$. Afterwards we evaluate the total $L_{2}$ error in each triangle. We insert a single mask pixel in each triangle, in descending order of the $L_{2}$ error. The position of the mask pixel within a triangle is chosen to be at the empty location with the largest pointwise error.

This procedure ensures that the inserted mask points are adapted to the evolving FEM mesh, which is the main advantage over previous approaches 8. 17]. Generally, the more iterations/inpaintings $n$ are allowed, the higher the quality of the mesh is for the purpose of approximating $f$. Practically useful numbers range between $n=10$ and $n=100$.

\section{Tonal Optimisation}

Let the grey values in the $m$ optimised mask pixels be given by a vector $\boldsymbol{g} \in \mathbb{R}^{m}$. Then the corresponding inpainting result $\boldsymbol{u}$ can be written formally as $\boldsymbol{u}=\boldsymbol{B} \boldsymbol{g}$, where the matrix $\boldsymbol{B}$ is dense and depends only on the mesh and the enumeration of the vertices. It also includes the discussed interpolation from Section 3 for image pixels that are not vertices in the mesh. The goal of the tonal optimisation problem is to find the grey values $\boldsymbol{g}^{*}$ that give the best reconstruction $\boldsymbol{u}^{*}=\boldsymbol{B} \boldsymbol{g}^{*}$ :

$$
\boldsymbol{g}^{*}=\underset{\boldsymbol{g} \in \mathbb{R}^{m}}{\arg \min }\|\boldsymbol{B} \boldsymbol{g}-\boldsymbol{f}\|_{2}^{2} .
$$

Since this is a linear least squares problem, its solution is given by the linear system of equations (the so-called normal equations):

$$
\boldsymbol{B}^{T} \boldsymbol{B} \boldsymbol{g}=\boldsymbol{B}^{T} \boldsymbol{f} .
$$

A straightforward approach to solve the normal equations would compute $\boldsymbol{B}$ explicitly. Unfortunately, this would require memory that scales quadratically in the number of pixels. This becomes unfeasible for very large images.

As a remedy, we exploit the fact that $\boldsymbol{B}$ is formed as the product of a sparse matrix (interpolation) and the inverse of a sparse matrix (Laplace equation). This means that the required memory for our optimisation scales linearly in the number of pixels. We achieve this by applying a nested conjugate gradient solver to (5). The inner iterations are effectively solving inpainting problems due to the Laplace equation instead of inverting the matrix from the FEM formulation explicitly. The outer iterations optimise the grey value vector $\boldsymbol{g}$.

Interestingly, our approach results not only in a better memory scaling: Also its runtime is much lower than the inpainting echo approach [21], and it is also faster than alternative tonal optimisation techniques [12,15] for usual mask densities.

\section{Experiments}

Figure 1 displays the results of our FEM approach for three classical test images that are popular for sparse harmonic inpainting: trui, walter, and (a $256 \times 256$ 
original image trui
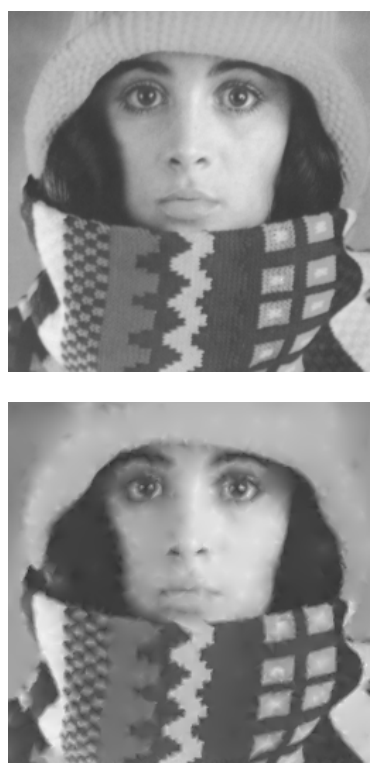

FEM reconstruction original image walter
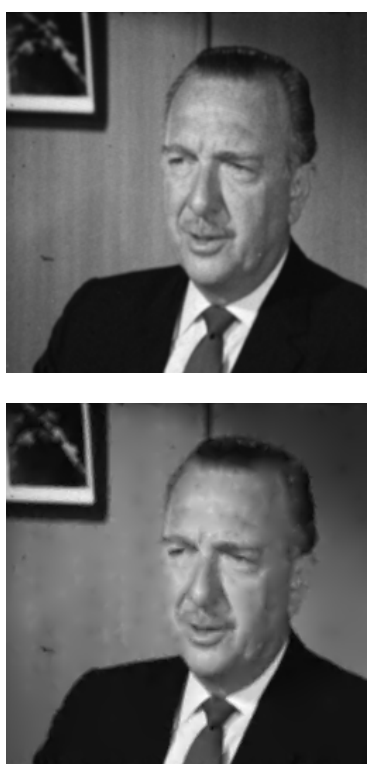

FEM reconstruction original image peppers
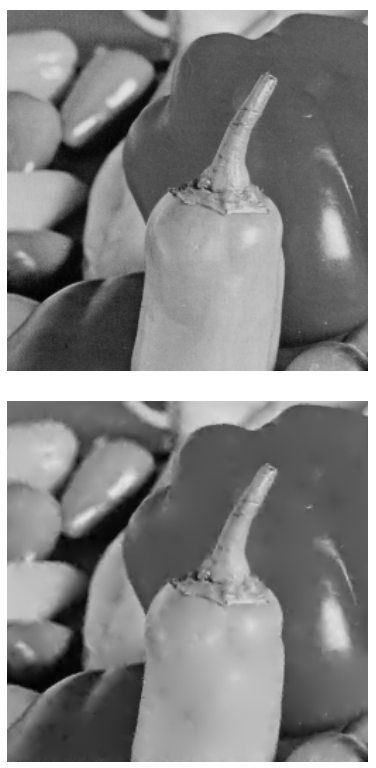

FEM reconstruction

Fig. 1. FEM reconstructions for trui, walter, and peppers with $4 \%$ density and $n=100$.

crop of) peppers. We see that even at a low mask density of $4 \%$, the reconstructions are fairly convincing. A quantitative analysis in terms of the mean squared error (MSE) is presented in Table 1. where we also compare our densification methods against the probabilistic sparsification results of Hoeltgen et al. 12. We observe that our algorithms achieve a consistently better quality. When we apply tonal optimisation after the spatial optimisation, the MSE decreases further by about one third. Sometimes tonal optimisation can even fully compensate suboptimal spatial optimisation, as can be seen for the peppers image.

Table 1. MSE comparisons at $4 \%$ density without and with tonal optimisation (TO).

\begin{tabular}{|c|c|c|c|c|c|c|}
\hline \multirow{2}{*}{ Method } & \multicolumn{2}{|c|}{ trui } & \multicolumn{2}{|c|}{ walter } & \multicolumn{2}{|c|}{ peppers } \\
\hline & no TO & with TO & no TO & with TO & no TO & with TO \\
\hline Prob. spars. $\left(q=10^{-6}\right)$ & 66.11 & 36.04 & 32.96 & 19.24 & 44.85 & 28.58 \\
\hline Ours $(n=10)$ & 44.62 & 30.07 & 19.09 & 12.62 & 43.20 & 29.83 \\
\hline Ours $(n=30)$ & 40.58 & 28.21 & 16.35 & 11.09 & 38.37 & 28.11 \\
\hline Ours $(n=100)$ & 37.60 & 26.62 & 15.92 & 11.21 & 36.68 & 28.85 \\
\hline
\end{tabular}




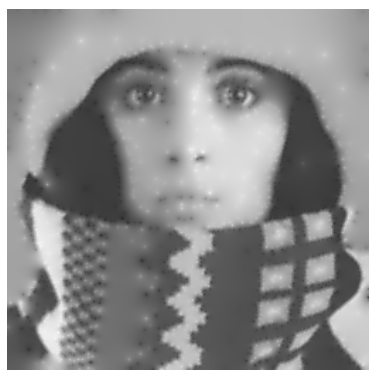

FDM, MSE $=36.04$

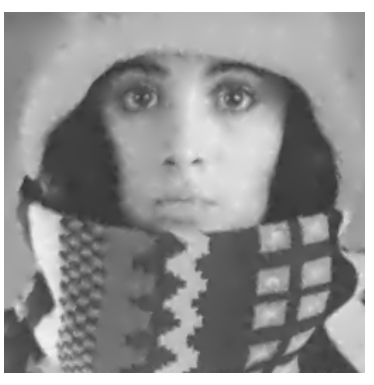

FEM, $\quad \mathrm{MSE}=26.62$

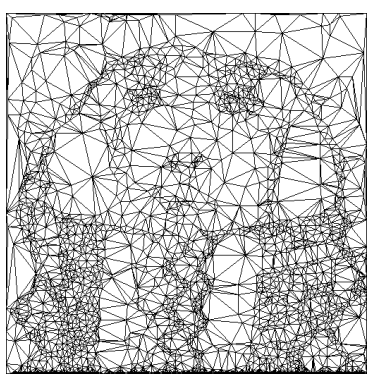

Delaunay triangulation

Fig. 2. Comparison between FDM with probabilistic sparsification (left) and FEM with densification (middle), both at $4 \%$ density and tonally optimised. The FEM result does not suffer from the pronounced singularities on the scarf and face present for FDM. Right: Delaunay triangulation used for the FEM method.

Table 2. Runtime scaling with resolution (in seconds) of our spatial optimisation and tonal optimisation with $n=10$ at a density of $4 \%$.

\begin{tabular}{lccccc}
\hline image size & $64 \times 64$ & $128 \times 128$ & $256 \times 256$ & $512 \times 512$ & $1024 \times 1024$ \\
\hline spatial optimisation & 0.01 & 0.03 & 0.11 & 0.49 & 2.07 \\
\hline tonal optimisation & 0.03 & 0.14 & 0.53 & 2.96 & 12.77 \\
\hline
\end{tabular}

In terms of runtime for the spatial optimisation, a probabilistic sparsification of a $256 \times 256$ image with the parameters from $[12$ on a Ryzen $4800 \mathrm{H} \mathrm{CPU}$ takes about 10 minutes, while our FEM densification needs only 0.3 seconds. This shows that our method is 1800 times faster. This factor grows rapidly with the image size: For $512 \times 512$ images, it is already 10,000 ( 4 hours versus 1.3 seconds). The complexity of the optimisation is $\mathcal{O}(n q \sqrt{k})$, where $n$ is the number of iterations, $k$ is the condition number of the matrix, $q$ is the number of non-zero entries in the matrix (at most $6 \times$ the number of vertices of the FEM mesh).

Our tonal optimisation algorithm is also several orders of magnitude faster, requiring $0.3 \mathrm{~s}$ for a $256 \times 256$ image. The reported runtimes from Hoeltgen et al. 12 on a Xeon $3.2 \mathrm{GHz}$ CPU vary between $77 \mathrm{~s}$ and $458 \mathrm{~s}$ for the different algorithms discussed there. The tonal optimisation has a time complexity of $\mathcal{O}\left(q \sqrt{k_{2}} \sqrt{k}\right)$, where $k_{2}$ is the condition number of the tonal optimisation matrix. Additionally, previous methods relying on QR or LU factorisations [14] require memory that grows quadratically in the number of mask pixels. In contrast, our algorithm's memory requirements grow linearly in the number of image pixels $(12 q+32 v$ bytes, where $v$ is the number of vertices in the FEM mesh). Thus, it can be applied to much larger images.

In Figure 2, we juxtapose FEM method with densification to the FDM approach with probabilistic sparsification, both in the tonally optimised case. We observe that the FEM technique does not suffer from the pronounced singulari- 


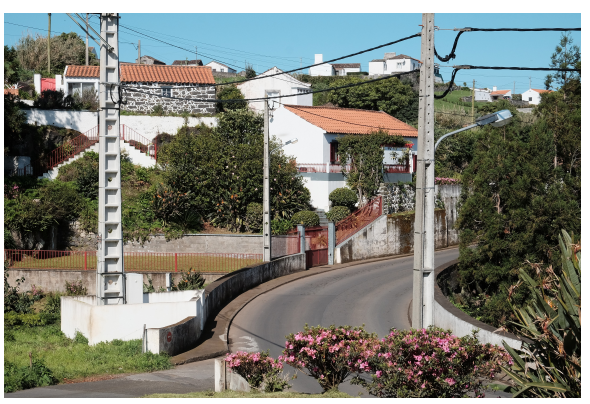

original image saomiguel

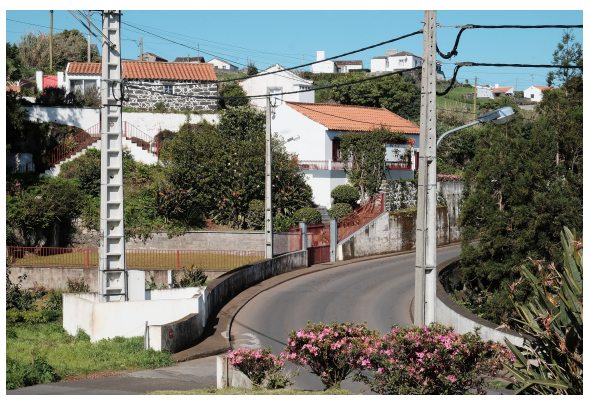

FEM reconstruction

Fig. 3. Left: A richly textured colour image of size $4896 \times 3264$ amounting to ca. 16 million pixels. Photo: J. Weickert. Right: Our FEM reconstruction with $n=30$ iterations, $10 \%$ mask density, and $L_{1}$ optimisation.

ties around mask pixels. These artifacts can be explained by the logarithmic singularities of the Green's functions in the continuous harmonic inpainting model; see 23 for more details. Our FEM approach with linear approximations within each triangle handles them more gracefully.

Table 2 reports scaling results for the spatial and tonal optimisation steps of our FEM method. We see that it scales almost linearly with the number of image pixels. The slight deviations from an ideal linear scaling are caused by the conjugate gradient solver: Large images lead to a higher condition number of the linear systems, such that the conjugate gradient method needs a few more iterations 28].

Finally, Figure 3 illustrates that our approach can also reconstruct large and richly textured colour images with very high perceptual quality. Extending the method to colour images is straightforward. In order to achieve highest visual fidelity, we have followed 31] and optimised the $L_{1}$ error instead of the $L_{2}$ one.

\section{Conclusions and Future Work}

Our paper is the first one that considers finite elements for inpainting-based image compression. We have seen that they offer a much higher efficiency due to their better adaptivity to the image structure. Moreover, our algorithms have substantially better scaling properties than previous approaches. This allows to apply them to much larger images, which is an important issue for bringing inpainting-based compression into practical applications.

In our ongoing work, we are studying options to increase the efficiency of our approach even further and to extend it to other PDEs and the compression of data on manifolds. 


\section{References}

1. Adams, M.: A highly effective incremental/decremental Delaunay mesh generation strategy for image representation. Signal Processing 93(4), 749-764 (Apr 2013)

2. Andris, S., Peter, P., Mohideen, R., Weickert, J., Hoffmann, S.: Inpainting-based video compression in FullHD. In: Elmoataz, A., Quéau, J.F.Y., Rabin, J., Simon, L. (eds.) Scale Space and Variational Methods in Computer Vision. Lecture Notes in Computer Science, Springer (2021), to appear

3. Bänsch, E., Mikula, K.: A coarsening finite element strategy in image selective smoothing. Computation and Visualization in Science 1, 53-61 (Jul 1997)

4. Bonettini, S., Loris, I., Porta, F., Prato, M., Rebegoldi, S.: On the convergence of a linesearch based proximal-gradient method for nonconvex optimization. Inverse Problems 33(5) (Mar 2017), article 055005

5. Boujena, S., Bellaj, K., Gouasnouane, O., Guarmah, E.: An improved nonlinear model for image inpainting. Applied Mathematical Sciences 9(124), 6189-6205 (Jan 2015)

6. Chen, Y., Ranftl, R., Pock, T.: A bi-level view of inpainting-based image compression. In: Kúkelová, Z., Heller, J. (eds.) Proc. 19th Computer Vision Winter Workshop. Křtiny, Czech Republic (Feb 2014)

7. Dai, Q., Chopp, H., Pouyet, E., Cossairt, O., Walton, M., Katsaggelos, A.: Adaptive image sampling using deep learning and its application on X-ray fluorescence image reconstruction. IEEE Transactions on Multimedia 22(10), 2564-2578 (Oct 2020)

8. Daropoulos, V., Augustin, M., Weickert, J.: Sparse inpainting with smoothed particle hydrodynamics. arXiv:2011.11289 [eess.IV] (Nov 2020)

9. Demaret, L., Dyn, N., Iske, A.: Image compression by linear splines over adaptive triangulations. Signal Processing 86(7), 1604-1616 (Jan 2006)

10. Distasi, R., Nappi, M., Vitulano, S.: Image compression by B-tree triangular coding. IEEE Transactions on Communications 45(9), 1095-1100 (Sep 1997)

11. Galić, I., Weickert, J., Welk, M., Bruhn, A., Belyaev, A., Seidel, H.P.: Towards PDE-based image compression. In: Paragios, N., Faugeras, O., Chan, T., Schnörr, C. (eds.) Variational, Geometric and Level-Set Methods in Computer Vision, Lecture Notes in Computer Science, vol. 3752, pp. 37-48. Springer, Berlin (Oct 2005)

12. Hoeltgen, L., Mainberger, M., Hoffmann, S., Weickert, J., Tang, C.H., Setzer, S., Johannsen, D., Neumann, F., Doerr, B.: Optimising spatial and tonal data for PDE-based inpainting. In: Bergounioux, M., Peyré, G., Schnörr, C., Caillau, J.P., Haberkorn, T. (eds.) Variational Methods in Imaging and Geometric Control, pp. 35-83. De Gruyter, Berlin (2017)

13. Hoeltgen, L., Setzer, S., Weickert, J.: An optimal control approach to find sparse data for Laplace interpolation. In: Heyden, A., Kahl, F., Olsson, C., Oskarsson, M., Tai, X.C. (eds.) Energy Minimisation Methods in Computer Vision and Pattern Recognition, Lecture Notes in Computer Science, vol. 8081, pp. 151-164. Springer, Berlin (Aug 2013)

14. Hoeltgen, L., Weickert, J.: Why does non-binary mask optimisation work for diffusion-based image compression? In: Tai, X.C., Bae, E., Chan, T.F., Leung, S.Y., Lysaker, M. (eds.) Energy Minimisation Methods in Computer Vision and Pattern Recognition, Lecture Notes in Computer Science, vol. 8932, pp. 85-98. Springer, Berlin (Jan 2015)

15. Hoffmann, S.: Competitive image compression with linear PDEs. Ph.D. thesis, Department of Computer Science, Saarland University, Saarbrücken, Germany (2016) 
16. Johnson, C.: Numerical Solution of Partial Differential Equations by the Finite Element Method. Dover, New York (2009)

17. Karos, L., Bheed, P., Peter, P., Weickert, J.: Optimising data for exemplar-based inpainting. In: Blanc-Talon, J., Helbert, D., Philips, W., Popescu, D., Scheunders, P. (eds.) Advanced Concepts for Intelligent Vision Systems, Lecture Notes in Computer Science, vol. 11182, pp. 547-558. Springer, Berlin (Sep 2018)

18. Kačur, J., Mikula, K.: Solution of nonlinear diffusion appearing in image smoothing and edge detection. Applied Numerical Mathematics 17, 47-59 (Mar 1995)

19. Liu, D., Sun, X., Wu, F., Li, S., Zhang, Y.Q.: Image compression with edge-based inpainting. IEEE Transactions on Circuits, Systems and Video Technology 17(10), 1273-1286 (Oct 2007)

20. Mainberger, M., Bruhn, A., Weickert, J., Forchhammer, S.: Edge-based compression of cartoon-like images with homogeneous diffusion. Pattern Recognition 44(9), 1859-1873 (Sep 2011)

21. Mainberger, M., Hoffmann, S., Weickert, J., Tang, C.H., Johannsen, D., Neumann, F., Doerr, B.: Optimising spatial and tonal data for homogeneous diffusion inpainting. In: Bruckstein, A.M., ter Haar Romeny, B., Bronstein, A.M., Bronstein, M.M. (eds.) Scale Space and Variational Methods in Computer Vision, Lecture Notes in Computer Science, vol. 6667, pp. 26-37. Springer, Berlin (2012)

22. Marwood, D., Massimino, P., Covell, M., Baluja, S.: Representing images in 200 bytes: Compression via triangulation. In: Proc. 25th IEEE International Conference on Image Processing. pp. 405-409 (Oct 2018)

23. Melnikov, Y.A., Melnikov, M.Y.: Green's Functions: Construction and Applications. De Gruyter, Berlin (2012)

24. Mitchell, A.R., Griffiths, D.F.: The Finite Difference Method in Partial Differential Equations. Wiley, Chichester (1980)

25. Ochs, P., Chen, Y., Brox, T., Pock, T.: iPiano: Inertial proximal algorithm for nonconvex optimization. SIAM Journal on Imaging Sciences 7, 1388-1419 (2014)

26. Preparata, F.P., Shamos, M.I.: Computational Geometry: An Introduction. Texts and Monographs in Computer Science, Springer, New York (1985)

27. Preußer, T., Rumpf, M.: An adaptive finite element method for large scale image processing. In: Nielsen, M., Johansen, P., Olsen, O.F., Weickert, J. (eds.) ScaleSpace Theories in Computer Vision, Lecture Notes in Computer Science, vol. 1682, pp. 223-234. Springer, Berlin (Sep 1999)

28. Saad, Y.: Iterative Methods for Sparse Linear Systems. SIAM, Philadelphia, second edn. (2003)

29. Schmaltz, C., Peter, P., Mainberger, M., Ebel, F., Weickert, J., Bruhn, A.: Understanding, optimising, and extending data compression with anisotropic diffusion. International Journal of Computer Vision 108(3), 222-240 (Jul 2014)

30. Shewchuk, J.: What is a good linear element? Interpolation, conditioning, and quality measures. In: Chrisochoides, N. (ed.) Proceedings of the 11th International Meshing Roundtable, IMR 2002, Ithaca, New York, USA, September 15-18, 2002. pp. 115-126 (Jan 2002)

31. Sinha, P., Russel, R.: A perceptually based comparison of image similarity metrics. Perception 40(11), 1269-1281 (2011)

32. Theljani, A., Belhachmi, Z., Kallel, M., Moakher, M.: A multiscale fourth-order model for the image inpainting and low-dimensional sets recovery. Mathematical Methods in the Applied Sciences 40(10), 3637-3650 (Dec 2016) 